\section{AUTUMN DIARY}

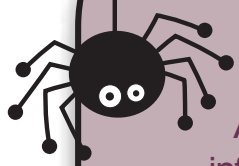

BDA DCP CPD events Assertiveness at work;

Achieving high standards in infection control (CORE);

Building a resilient dental team and reducing stress; Business planning and financial management; Child

Protection: the dental team's responsibility; Employment law rights and responsibilities; Growing and promoting your practice;

Handling complaints and improving communication skills (CORE);

IRMER course in dental radiography and radiation protection (CORE);

Law, ethics and record keeping (CORE); Management of medical emergencies for the whole dental team (CORE); Maximise your team's potential; Preparing for the $\mathrm{CQC}$ registration process; Reception and telephone skills for the whole team;

Risk management and clinical auditing; Treatment planning in the dental practice

Date: Ongoing

Telephone: 02075634590

Website: www.bola.org/events/training

London Deanery

An introduction to crisis management during medical emergencies

Free of charge;

maximum 8 per session

3 hours' verifiable CPD

Date: every first and third Wednesday of each month, 9am-12 noon or 1-4pm Email:sue.waller@

londondeanery.ac.uk

Training day for oral health promoters

Date: 30 September 201

Venue: Ramada Hote

Tunbridge Wells.

Email: wendy.mcevoy @nhs.net

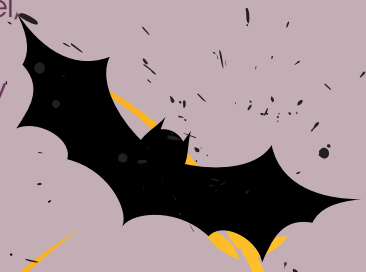

Hands-on photography workshop with Laura Horton

Date: 5 October 2011

Location: Leicester

Email: laura@

laurahortonconsulting.co.uk www.laurahortonconsulting.co.uk

Treatment Coordination workshop with Laura Horton

Date: 7 October 2011

Location: Leicester

Email: laura@

laurahortonconsulting.co.uk www.laurahortonconsulting.co.uk

West of Scotland Open Events for Dental Care Professionals

Date: 9 October 2011 10am-2pm/12

October 2011 5.30-8pm

Venue: West of Scotland Postgraduate Centre for Dental Education

Email: margaret.saunderson

@nes.scot.nhs.uk or katrina.

macfarlane@nes.scot.nhs.uk

Telephone: 01413522855

or 01413522856

\section{BDTA Dental Showcase}

Date: 20-22 October 2011

Venue: NEC, Birmingham

www.dentalshowcase.com

Hands-on restorative course for registered dental therapists and dental hygiene/therapists

Date: 26 October 2011

Venue: DCP School, Chapter House, Bristol Dental Hospital

Email: claire.price@uhbristol.nhs.uk Telephone: 01173424360

Bury Dental Education and Learning Set

Clinical Audit for the Dental Team

Date: 27 October 2011 Venue: Learning and Development Centre, Talbot Grove, Bury

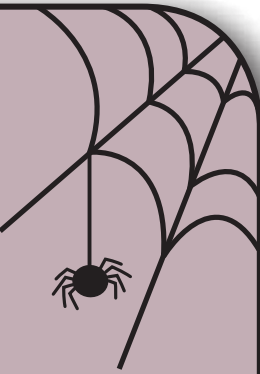

Email: samantha.ball@bury.nhs.uk Telephone: 01617671432 www.nwpgmd.nhs.uk

British Association of Dental Nurses (BADN) 2011 National Dental Nursing Conference Date: 11-12 November 2011

Venue: Glasgow Marriott

http://badn.org.uk/conference/

British Society of Dental Hygiene \& Therapy (BSDHT) Oral Health Conference \& Exhibition

Date: 18-19 November 2011

Venue: Bournemouth International Conference Centre

www.bsdht.org.uk

Dental Protection Practice Management Seminars

Date and location:

15 November 2011 (Leeds),

30 November 2011 (Edinburgh)

Telephone: 02073992914

Email: nicola.photiou@mps.org.uk

Premier Symposium 2011

Date: 26 November 2011

Venue: Imperial College, London

Telephone: 02073992914

Email: events@dentalprotection.org

Faculty of General Dental

Practice (UK) Scotland study day

Successful bleaching strategies

Date: 2-3 December 2011

Location: Glasgow

www.fgdp-scotland.org
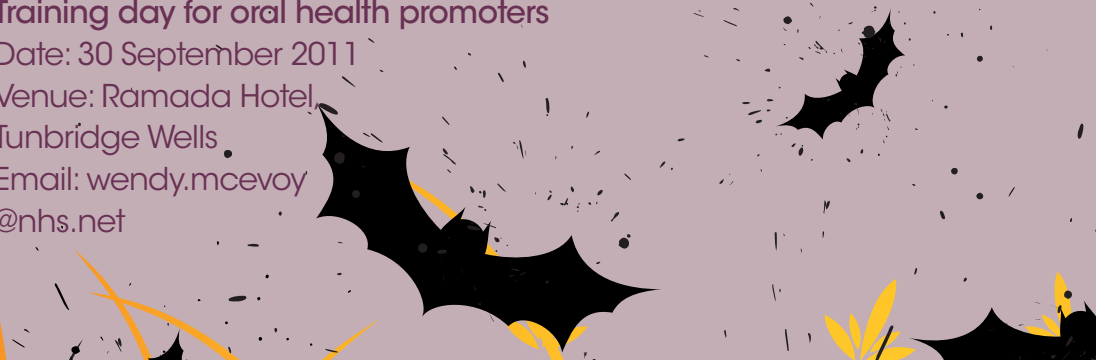